\title{
A NOTE ON IDEALS OF OPERATORS
}

\author{
RICHARD I. LOEBL
}

\begin{abstract}
An ideal $\mathscr{I}$ of $\mathscr{L}(\mathscr{H})$ is said to be multiplicatively prime if $A X B \in \mathscr{I}$ for all $X \in \mathscr{L}(\mathscr{H})$ implies $A$ or $B$ is in $\mathscr{I}$. The only normable multiplicatively prime ideals are $\{0\}$ and $\mathscr{K}$, the compacts. Multiplicative primeness is related to other properties an ideal may possess.
\end{abstract}

Considering the following properties that an ideal $\mathscr{I}$ of operators on separable Hilbert space may possess. (Here, ideal means two-sided and selfadjoint, but not closed.)

DEFINITION 1. An ideal $\mathscr{I}$ has the square root property if, for all $A \in \mathscr{I}$, we have $\sqrt{|A|} \in \mathscr{I}$. (As usual $|A|=\sqrt{A^{*} A}$.)

We note that $A \in \mathscr{I}$ if and only if $|A| \in \mathscr{I}$, by polar decomposition [4, p. 69].

Definition 2. An ideal $\mathscr{I}$ is square if $\mathscr{I}=\mathscr{I}^{2}$, i.e., every element $A \in \mathscr{I}$ can be written $A=B C$ where $B$ and $C$ are in $\mathscr{I}$.

Definition 3. An ideal $\mathscr{I}$ is multiplicatively prime if $A X B \in \mathscr{I}$ for all $X \in \mathscr{L}(\mathscr{H})$ implies $A$ or $B$ is in $\mathscr{I}$.

We remark that if $\operatorname{dim} \mathscr{H} \geqslant 2$, no proper two-sided ideal in $\mathscr{L}(\mathscr{H})$ is prime in the classical algebraic sense, i.e, $A B \in \mathscr{I}$ implies $A$ or $B$ in $\mathscr{I}$; just let

$$
A=\left[\begin{array}{ll}
A_{1} & 0 \\
0 & X
\end{array}\right] \text { and } B=\left[\begin{array}{cc}
Y & 0 \\
0 & B_{1}
\end{array}\right]
$$

where $A_{1}, B_{1}$ are in $\mathscr{I}$, but $X$ and $Y$ are not. For finite dimensions, $\mathscr{I}=\{0\}$; for infinite dimensions, it is an easy argument using s-numbers and ideal sets, cf. below, to see that $X$ not in $\mathscr{I}$ implies $A_{1} \oplus X$ not in $\mathscr{I}$.

It is evident that the improper ideal $\mathscr{L}(\mathscr{H})$ satisfies all three properties. We intend to find all the others. As a preliminary, we note that every proper two-sided ideal in $\mathscr{L}(\mathscr{H})$ is a subset of $\mathscr{K}$, the ideal of compact operators. For a compact operator $T$, the sequence of $s$-numbers of $T, s(T)$, is defined as follows: Let $\alpha_{1} \geqslant \alpha_{2} \geqslant \alpha_{3} \geqslant \cdots$ be the eigenvalues of $|T|$; then $s(T)=\left\{s_{n}(T)=\alpha_{n}\right\}=s(|T|)$.

An ideal set $I$ is a collection of sequences of real numbers $\left\{a_{n}\right\}_{n=1}^{\infty}$ with the following properties:

(i) if $\left\{a_{n}\right\} \in I$, then $a_{n} \geqslant 0$ for all $n$, and $\lim _{n} a_{n}=0$;

(ii) if $\left\{a_{n}\right\} \in I$ and $\pi$ is any permutation of the positive integers then $\left\{a_{\pi(n)}\right\} \in I$;

(iii) if $\left\{a_{n}\right\} \in I$ and $\left\{b_{n}\right\} \in I$, then $\left\{a_{n}+b_{n}\right\} \in I$;

(iv) if $\left\{a_{n}\right\} \in I$ and $0 \leqslant b_{n} \leqslant a_{n}$ for all $n$, then $\left\{b_{n}\right\} \in \mathscr{I}$.

Received by the editors September 10, 1984 and, in revised form, February 4, 1985. 1980 Mathematics Subject Classification. Primary 47D25. 
It is a classical result of Calkin [2] that there is a bijection between ideal sets and proper two-sided ideals of operators. We also have the following [3, Lemma 1.1], see also [2]:

Lemma. Let $\mathscr{I}$ be a proper two-sided ideal of $\mathscr{L}(\mathscr{H})$. A compact operator $T$ belongs to $\mathscr{I}$ if and only if $s(T)$ belongs to the ideal set of $\mathscr{I}$.

An ideal $\mathscr{I}$ is said to be a norm ideal if there is a norm $\|\cdot\|_{\mathscr{I}}$ on $\mathscr{I}$ with the following properties:

(i) $\left(\mathscr{I},\|\cdot\|_{\mathscr{I}}\right)$ is a Banach space;

(ii) $\|S T R\|_{\mathscr{I}} \leqslant\|S\|\|T\|_{\mathscr{I}}\|R\|$ for all $R, S \in \mathscr{L}(\mathscr{H})$, for all $T \in \mathscr{I}$;

(iii) $\|T\|_{\mathscr{J}}=\|T\|$ for $T$ of rank one.

The canonical examples of norm ideals are the Schatten ideals $C_{p}, 1 \leqslant p \leqslant \infty$, where $\|T\|_{p}$ is the $l^{p}$ norm of $s(T)$.

THEOREM 1. Consider the following properties of an ideal $\mathscr{I}$ :

(1) $\mathscr{I}$ is multiplicatively prime;

(2) I has the square root property;

(3) $\mathscr{I}$ is square.

Then $(1) \Rightarrow(2) \Leftrightarrow(3)$.

Proof. (1) $\Rightarrow$ (2). Suppose $A \in \mathscr{I}$; hence $|A| \in \mathscr{I}$. Look at the maping $X \rightarrow \sqrt{|A|} X$ $\sqrt{|A|}$ for $X \in \mathscr{L}(\mathscr{H})$. We claim the range of this mapping is in $\mathscr{I}$; if so, then by the property of being multiplicatively prime, we have $\sqrt{|A|} \in \mathscr{I}$.

But $X \rightarrow C X D$ has range in $\mathscr{I}$ if and only if $s(C) s(D)$ belongs to $I$, the ideal set of $\mathscr{I}$, by [3, Lemma 5.4 and 5.5]. Then $s(\sqrt{|A|}) s(\sqrt{|A|})=s(|A|) \in I$ by our earlier lemma, completing the proof.

(2) $\Rightarrow$ (3). The polar decomposition writes $A=U|A|=(U \sqrt{|A|}) \sqrt{|A|}$ as a product of elements in $\mathscr{I}$.

(3) $\Rightarrow$ (2). This follows from Theorem 2.1 of [6].

REMARK. Let $\mathscr{I}_{p}=\bigcup_{K \geqslant 0} C_{p 2^{K}}$ where $p \geqslant 1$; then $\mathscr{I}$ has the square root property, for if $\sum\left|\beta_{i}\right|^{r}<\infty$, then $\Sigma\left(\sqrt{\left|\beta_{i}\right|}\right)^{2 r}<\infty$; thus if $A \in C_{r} \subseteq \mathscr{I}_{p}$ then $\sqrt{|A|} \in C_{2 r} \subseteq \mathscr{I}_{p}$.

On the other hand, $\mathscr{I}$ is not multiplicatively prime, since there are compact operators $A$ and $B$ such that $A, B \notin C_{p}$ for every $p, 1 \leqslant p<\infty$, but such that $X \rightarrow A X B$ takes values in $C_{1}$ [3, Example 5.8].

Proposition 2. The ideals $0, \mathscr{F}$, and $\mathscr{K}$ are multiplicatively prime.

Proof. For $\mathscr{K}$, this is Proposition 4.1 of [3].

If $A X B=0$ for all $X$ with $A, B \neq 0$, let $u$ be so that $B u=v \neq 0$; and let $w$ be so that $A w \neq 0$. Then for $X$ the rank-one operator $X(e)=\langle e, v\rangle w$, we have $(A X B)(u)$ $\neq 0$.

If $A X B$ is finite rank for all $X$, but $A, B$ are not finite rank, choose $\left\{e_{i}\right\}_{i=1}^{\infty}$ such that $\left\{B e_{i}\right\}_{i=1}^{\infty}$ is an orthonormal basis for the range of $B$; and choose $\left\{f_{i}\right\}_{i=1}^{\infty}$ an orthonormal set so that $\left\{A f_{i}\right\}$ is a basis for the range of $A$. Then for the partial isometry $X: B e_{i} \rightarrow f_{i}$, we have $A X B$ is not finite rank. 
TheOREM 3. If $\mathscr{I}$ is a proper norm ideal different from $\mathscr{L}(\mathscr{H})$, the following are equivalent:

(1) $\mathscr{I}$ is multiplicatively prime,

(2) I has the square root property,

(3) $\mathscr{I}$ is square,

(4) $\mathscr{I}=\{0\}$ or $\mathscr{I}=\mathscr{K}$.

Proof. By Theorem 1 and Proposition 2, the only implication we need to prove is $(3) \Rightarrow(4)$. But this is precisely the content of [7, Theorem 2.9].

REMARKS. The above results lend to the following question: Which ideals $\mathscr{I}$ of $\mathscr{L}(\mathscr{H})$ are multiplicatively prime?

To see if $\mathscr{I}$ is multiplicatively prime, we ask whether $A X B \in \mathscr{I}$ for all $X \in \mathscr{L}(\mathscr{H})$ implies that $A$ or $B$ is in $\mathscr{I}$.

From [3, Lemma 5.1 and Corollary 5.2], we obtain the facts that if either $A$ or $B$ is not compact the only way for $A X B$ to be in $\mathscr{I}$ for all $X$ in $\mathscr{L}(\mathscr{H})$ is for $B$ (for $A$ respectively) to be in $\mathscr{I}$.

From Theorem 3, we see that norm ideals cannot be square, and thus cannot be multiplicatively prime. This also follows from Theorem 7.11 in [7], which states that if $\mathscr{I} \subsetneq \mathscr{K}$ is any norm ideal, there are ideals $\mathscr{I}_{1}, \mathscr{I}_{2}$ with $\mathscr{I} \subsetneq \mathscr{I}_{k}$ and $\mathscr{I}=\mathscr{I}_{1} \cap \mathscr{I}_{2}$. If we choose $A_{k} \in\left(\mathscr{I}_{k} / \mathscr{I}\right)$, then we have for all $X$ in $\mathscr{L}(\mathscr{H})$ that $A_{1} X A_{2} \in \mathscr{I}_{1} \cap \mathscr{I}_{2}=$ $\mathscr{I}$. From $[3,5.4]$ we have $s\left(A_{1}\right) s\left(A_{2}\right) \in I$, but $s\left(A_{i}\right) \notin I$ since $A_{i} \notin \mathscr{I}$.

We conjecture that, among nonnorm ideals, only $\mathscr{F}$ is multiplicatively prime.

\section{REFERENCES}

1. A. Brown, C. Pearcy and N. Salinas, Ideals of compact operators on Hilbert space, Michigan Math. J. 18 (1971), 373-384.

2. J. W. Calkin, Two-sided ideals and congruences in the ring of bounded operators in Hilbert space, Ann. of Math. (2) 42 (1971), 839-873.

3. L. Fialkow and R. Loebl, Elementary mappings into ideals of operators, Illinois J. Math. 28 (1984), 555-578.

4. P. R. Halmos, A Hilbert space problem book, Van Nostrand, New York, 1967.

5. N. Salinas, Ideal sets and ideals of compact operators, Indiana Univ. Math. J. 22 (1972), 505-521.

6. __ Ideals of commutators of compact operators, Acta Sci. Math. Szeged 36 (1974), 131-144.

7. $213-240$.

Department of Mathematics, Wayne State University, Detroit, Michigan 48202 